\title{
Una revisión narrativa: el baloncesto como medio de inclusión en el trastorno del espectro autista \\ A narrative review: basketball as a mean of inclusion for people with autistic spectrum disorder \\ *Elisa García-Obrero, **Higinio González-García \\ *Universidad Complutense de Madrid (España), **Universidad Internacional de la Rioja (España)
}

\begin{abstract}
Resumen: Actualmente se encuentran en auge los estudios que relacionan el Trastorno del Espectro Autista con la actividad física, siendo el deporte sinónimo de mejora en la calidad de vida de las personas. Sin embargo, todas las investigaciones que unifican elTrastorno del Espectro Autista y deporte quedan asociadas a prácticas individuales, como la natación o la equitación. El objetivo de la revisión narrativa es conocer qué suscita la literatura en relación al TEA con el deporte, concretando en los deportes colectivos y especificando en la práctica de baloncesto. La presente revisión narrativa reflexiona abrir la oferta deportiva hacia deportes colectivos, concretamente el baloncesto, encontrando en su práctica un escenario muy positivo y beneficioso para el desarrollo integral de las personas con TEA. Se acredita el deporte como medio indispensable de inclusión y crecimiento de las personas con TEA, además de evidenciarse el potencial de los deportes colectivos, concretamente el baloncesto, a la hora de progresar en aspectos diagnósticos donde las personas con TEA muestran más dificultades (relaciones sociales, funciones ejecutivas, relación en y con el entorno). Como conclusiones, se sugiere la necesidad de continuar con investigaciones que asocien elTEA a deportes colectivos, además de trabajos de campo que tengan como objeto de estudio el baloncesto y ayuden así a la producción de conocimiento entorno a las posibilidades inclusivas y de desarrollo que ofrece su práctica para las personas con TEA.
\end{abstract}

Palabras clave: Trastorno del Espectro Autista; deporte; baloncesto; inclusión.

\begin{abstract}
The studies that relate the Autism Spectrum Disorder with the physical activity have currently increased, being sport a byword for improvement on people's life quality. However, all the investigations that join the Autism Spectrum Disorder and sport refer to individual performances, such as swimming or equestrianism. Thus, the goal of the narrative review is to recognize what produces the literature about Autism Spectrum Disorder and sports (focusing on group sports, particularly basketball). This narrative revision suggests an ample sport offer in collective sports, especially basketball, finding on its practice a very positive and beneficial set for the absolute development of people with ASD. Sport is ratified as an indispensable channel of inclusion and growth in people with autism, in addition to evince the potential of group sports, specifically basketball, when progressing in diagnostic aspects where people with ASD find more difficulties (social relationships, executive functions, relationship with and in the environment). As conclusions, it is highlighted the need of continuing with investigations that relate autism with group sports, as well as field studies regarding basketball producing this way knowledge about the possibilities of inclusion and development that its practice offers to people with ASD.
\end{abstract}

Keywords: Autism Spectrum Disorder; sport; basketball; inclusion.

\section{Introducción}

Las palabras «discapacidad»y «deporte» han sido términos desconectados a lo largo de la historia. Su práctica no parece actualmente facilitar su inclusión, pues no se permite su plena participación (Robles-Rodríguez, Abad-Robles, Giménez \& Benito-Peinado, 2017). El deporte es un fenómeno social que tiene la posibilidad de mejorar el estado emocional, el desarrollo personal y social de las personas con discapacidad (Muñoz, Ga-

Fecha recepción: 28-10-20. Fecha de aceptación: 15-05-21

Elisa García Obrero

elisag11@ucm.es rrote \& Sánchez, 2017). Sin embargo, la práctica físicodeportiva engloba en sí misma barreras tales como ausencia de implicación institucional, falta de accesibilidad -cognitiva y espacial-, carencia de profesionales con la formación pertinente e inadecuación de material adaptado -bien por el alto coste económico o por las dificultades de ser posibilitado- (Pérez-Tejero, Ocete, Ortega-Vila \& Coterón, 2012). Además, aún siguen vigentes prejuicios basados en creencias negativas hacia las personas con discapacidad, siendo la base de actitudes negativas (discriminación, distanciamiento, indiferencia, negación de ayuda o faltas de respeto) que suponen una auténtica lacra en el camino hacia la inclusión social y deportiva (Felipe, Garoz \& Tejero, 2018).

Actualmente, se reconoce el derecho de toda per- 
sona a la práctica deportiva sin ser excluido por motivos de género, económicos, sociales o cualquier tipo de discapacidad (Ramírez, Negrete, Díaz, Rubiano \& Hernández, 2016). La inclusión deportiva no sólo puede, sino que debe girar alrededor de los derechos de las personas con discapacidad (Asenjo \& Caro, 2019). Sin embargo, no parece que el deporte sea una vía valorada como herramienta normalizadora y de inclusión social de primer orden (Pérez-Tejero et al., 2012) a pesar de ser un gran medio para favorecer la comunicación, socialización y autonomía personal, desarrollar destrezas cognitivas, promocionar la salud y mejorar la capacidad física y motriz (Klein, 2019).

En el caso de las personas conTrastorno del Espectro Autista (en adelante TEA), la práctica físico-deportiva juega un papel crucial en el desarrollo y funcionamiento físico, fisiológico, psicológico y conductual de los individuos con dicho trastorno (Latorre, Sánchez, Salas \& García, 2018). Existen evidencias de la reducción de conductas repetitivas y estereotipadas a través de la práctica deportiva, sumado este hecho a los numerosos beneficios que supone el deporte como mejora física y fisiológica, aumento de la autoestima y reducción de estrés y ansiedad, atendiendo al aspecto psicológico (Sánchez, Sánchez, Pastor \& Martínez, 2019).

La autosuperación es la esencia de la práctica deportiva de las personas con discapacidad (Asenjo \& Caro, 2019). Ese afán debe ser contagiado a toda la sociedad para, por un lado, reconocer todos los beneficios de la práctica deportiva y, por otro lado, cooperar de manera conjunta para no obviar la actividad física y el deporte como medio no sólo de mejora de diagnóstico, sino como espacio de disfrute y aprendizaje para todas las personas. Si bien la inclusión es comprendida originalmente desde los términos de comunidad y participación, la actividad-física cumple el desarrollo de la persona en sociedad: propositiva, autónoma, proactiva, consciente y reflexiva -desde el hacer, pensar y actuar- (Fernández \& Camargo, 2021).

El presente trabajo analiza de manera exhaustiva el rol del deporte a lo largo de la historia desde la perspectiva de la diversidad, incidiendo de manera especial en la relación del deporte con el TEA. Otro de los aspectos a profundizar son las características del TEA, estableciendo las bases para conocer su relación con el deporte y, finalmente, su pretendido nexo al baloncesto. Para ello, se estableció el siguiente objetivo general: revisar qué dicta la literatura en relación al TEA con el deporte, añadiendo el baloncesto como deporte a examinar, dada su posible potencialidad en el desarrollo integral de personas con TEA. Por otro lado, con el fin de responder al objetivo general, se establecieron los siguientes objetivos específicos:

- Comprobar el rol del deporte y su inclusión en las personas con diversidad funcional, concretando en el TEA.

- Conocer las características que definen actualmente el TEA.

- Valorar los deportes colectivos como herramienta potencial en el desarrollo integral de las personas con TEA, concretando en el baloncesto.

\section{Metodología}

Para la elaboración del trabajo se lleva a cabo una revisión narrativa de la literatura científica en relación alTEA, el deporte y la vinculación al baloncesto. En la búsqueda se han tenido en cuenta artículos escritos en castellano; a excepción de un artículo escrito en inglés (Massion, 2006) y uno en portugués (Braga, 2011), obtenidos de las referencias de los artículos en castellano. Las palabras clave que vertebran la esencia conceptual del texto son «deporte», «Trastorno del Espectro Autista», «inclusión», «baloncesto»y «deportes colectivos». Además, en el apartado «progresión histórica del baloncesto en materia de inclusión» se tiene en cuenta un vídeo-documental para la redacción del mismo (Barthe, 1992). Los artículos se encuentran localizados en las bases de Dialnet, Scopus, Google Scholar, Redalyc y SciELO, tratando de valorar sólo artículos de actualidad (publicaciones en los últimos diez años, es decir, desde 2011). Encontrándose como excepción en seis artículos, debido al escaso desarrollo del año 2011 (la revisión se redacta entre diciembre 2020-enero 2021; 4 de los 7 artículos que exceptúan la norma son del año 2010 y 2006). Como se comentó anteriormente, una vez se hicieron las búsquedas, de los artículos encontrados también se obtuvieron algunos trabajos, a través de las referencias de dichas investigaciones. Estos artículos que se incluyeron de las referencias, no se encontraban disponibles dentro de las bases de datos buscadas, y se seleccionaron debido a su gran relación y relevancia con la temática.

\section{El autismo y el niño}

La discapacidad es un concepto que tiene sus orígenes en la medicina, surge ante la necesidad de unir los distintos trastornos en una misma terminología para erradicar palabrería y actitudes peyorativas como re- 
tardo, inválido o incapacitado (Sánchez et al., 2019). En este sentido, se calcula que las personas con discapacidad son el 15\% de la población total (Sánchez et al., 2019). Por otro lado, el TEA está caracterizado por anomalías en las interacciones sociales, deficiencias en la comunicación y el lenguaje, comportamientos estereotipados e intereses restrictivos y repetitivos (DSM-V, 2013). En España, el índice de personas con autismo es uno por cada 150 nacimientos (Sánchez et al., 2019). A nivel mundial, podemos hablar de una persona con TEA por cada 68. Además, se está viviendo un incremento durante los últimos años de personas diagnosticadas con TEA (André, Valdez, Ortiz \& Gámez, 2020).

Siguiendo el DSM-V (2013), elTrastorno del Espectro Autista queda subdividido en tres apartados: el Trastorno Autista, el Síndrome de Asperger y el Trastorno Generalizado del Desarrollo no especificado (Suárez, López, Belchior \& Dacruz, 2019). Pueden reconocerse distintas formas de autismo o trastornos de la misma naturaleza y similitud, es decir, puede manifestarse de manera muy diferente entre las personas con la misma patología (Heredia \& Duran, 2013). Por este motivo, hay que actuar de manera distinta con cada persona con autismo (Sánchez et al., 2019). Actualmente, derivado de la forma de categorizar los trastornos, se persigue el desarrollo en los valores fundamentales de la vida de las personas, evitando que los esfuerzos sean orientados a luchar contra la sintomatología (Lozano, Manzano, Casiano \& Aguilera, 2017).

Es importante facilitar ambientes muy ordenados con mucha estimulación, utilizar directrices claras, participar en variedad de actividades y evitar las ayudas excesivas (Heredia \& Duran, 2013). Es fácil que las personas con TEA aboguen por la soledad, encierro en las tecnologías y tareas solitarias que evadan las relaciones sociales. Parte del gusto por el ocio sedentario viene fomentado por las familias, quienes encuentran en las tecnologías una herramienta de distracción para los niños y las niñas con autismo (Ruiz et al., 2015).

\section{El papel del deporte en una sociedad inclusiva}

\section{Importancia del deporte en el desarrollo perso- nal y social}

El desarrollo integral de las personas a través del deporte no es una ficción, la ciencia demuestra que son muchos los beneficios generados (Braga, 2011; Sánchez \& Collado, 2016). Existen cinco ejes de interés que motivan a las personas a realizar deporte: disfrute, apa- riencia, salud, competencia y el ámbito social (Moreno, Águila \& Borges, 2011).

Desde el punto de vista social, el juego, la actividad física y el deporte son aliados directos con la educación en todos los contextos; los motivos son claros: el carácter vivencial y lúdico de la práctica deportiva, su potencialidad en aspectos como la cooperación y superación, las interacciones personales generadas y la aparición continua de conflictos a resolver (Rello \& Garoz, 2014). Si se prefiere verlo desde otra perspectiva sin perder el foco social, puede hablarse del deporte como prevención de conductas de riesgo, pues genera habilidades, actitudes y hábitos saludables que producen en las personas comportamientos prosociales; además, colabora en el autocontrol, tolerancia a la frustración, consideración y respeto hacia los demás, y ayuda a la estabilidad emocional (Pelegrín, Garcés \& Cantón, 2010). El proceso de socialización en el deporte se agrupa en tres subapartados: socialización dentro del deporte, a través del deporte y fuera del deporte (Pelegrín et al., 2010); todos son contribuyentes activos de la meta de afiliación social que persigue la actividad deportiva, transferible a la vida cotidiana (Moreno, Águila \& Borges, 2011).

En referencia al disfrute, es un rasgo motivacional en la personalidad de las personas, generadora de esquemas que confrontarán, en mayor o menor medida, su realidad vital o manera de interactuar con el entorno físico-social (Chamero \& Fraile, 2013). La relación de los factores afectivo-emocionales con el deporte tienen su foco de relevancia en la niñez, pues si la persona asocia realizar deporte a una sensación positiva, feliz y agradable, es más probable que alargue su práctica en el tiempo (Chamero \& Fraile, 2013). Es vital crear un espacio de confianza donde los niños y las niñas que realizan deporte se sientan contentos, valorados, con confianza y motivación (Carbonero \& Prat, 2012).

Atendiendo a la parte física, el deporte es una gran fuente de salud (Caracuel \& Arbinaga, 2012). En este sentido, Caracuel \& Arbinaga (2012) confirman que la actividad físico-deportiva atrae los siguientes beneficios, siempre y cuando su práctica sea de carácter estable en el tiempo y de media-larga duración: mejora en el funcionamiento del sistema locomotor; prevención, mejora y rehabilitación de enfermedades cardiovasculares; mejora en dolencias pulmonares, enfermedades cardiovasculares y dolencias crónicas como la diabetes o lesiones deportivas; ayuda en el control del peso. Estos datos son claves especialmente en la actualidad, donde existe una situación alarmista ante la población infantil por el aumento significativo de personas con sobrepeso, 
obesidad y ligadas a un ocio sedentario (Chamero \& Fraile, 2013).

El tono muscular, regulado con la formación reticular, está íntimamente ligado a los procesos de atención. Existe un vínculo directo entre la actividad tónica muscular y la actividad tónica cerebral (Fernández, 2011). Por ello, se puede ver la relación en el beneficio físico-psicológico-social (Moreno, Águila \& Borges, 2011) o, en otras palabras, el bienestar psicológico asociado a la práctica deportiva (Caracuel \& Arbinaga, 2012). Por otro lado, el deporte influye en la autopercepción física, pues proporciona novedosas experiencias corporales, enaltece la percepción de logros físicos en las personas, redefine las capacidades físicas y aumenta su confianza en la práctica de actividades físico- deportivas (Pérez-Tejero et al., 2012).

Tal es la importancia del deporte que las instituciones políticas del Estado en España tienen, de obligado cumplimiento, un compromiso con ello, tal como marca la Constitución Española (1978) en su artículo 43.3: «los poderes públicos fomentarán la educación sanitaria, la educación física y el deporte. Asimismo, facilitarán la adecuada utilización del ocio» (Asenjo \& Caro, 2019). La adecuación es fundamental, pues los factores ambientales actúan aumentando o frenando las posibilidades de la práctica físico- deportiva (Tejero, De laVega, Vaquero \& Ruiz, 2016).

A través del deporte los niños y niñas ayudan a su desarrollo físico, psíquico y social, configuran su carácter a través de experiencias, establecen hábitos y crean preferencias (Carbonero \& Prat, 2012). La actividad deportiva y los cánones de comportamiento transmitidos ayudan de manera total a la construcción de un gran ambiente socializador y la adquisición de valores como el respeto, la tolerancia y el juego limpio (Moscoso \& Muñoz, 2012).

\section{Deporte y autismo}

El ámbito deportivo supone uno de los indicadores más obvios en relación con el grado de cumplimiento de la inclusión, por parte de la sociedad, de las personas con diversidad funcional (Asenjo \& Caro, 2019). Existen cada vez más organizaciones que fomentan la práctica deportiva de las personas con discapacidad (Asenjo \& Caro, 2019). Este hecho es en gran manera reseñable, pues hasta hace pocos años el deporte no había sido apenas reconocido para las personas con discapacidad (Sánchez et al., 2019). Los programas deportivos suponen una mejoría en la calidad de vida (Ramírez et al., 2016), además de tener la capacidad de disminuir facto- res de riesgo en enfermedades y generar variaciones positivas en mejora de la salud (Santos, Jiménez \& Rioja, 2019). Otro aspecto muy beneficioso es la evidencia de la práctica deportiva como medio potente de inclusión en relación con el contexto, por medio de la mejora en la regulación emocional y estado de ánimo generado por la actividad física (Bernate, Rojas, Fonseca \& Betancourt, 2020). Las emociones no siempre han estado valoradas como parte fundamental del ser humano, si bien en la actualidad son consideradas un componente esencial (Mujica, 2018). Las emociones suponen un proceso multidimensional donde se interpretan subjetivamente situaciones significativas y la expresión o comunicación de emociones, atendiendo a un proceso con componentes experienciales, observacionales-motores y fisiológicos del sujeto que las retiene (Reyna, 2011). La emoción es la respuesta de un proceso complejo mental (Mujica, 2018), lo cual para las personas con autismo supone un reto mayor debido, entre otros, a su dificultad para las relaciones sociales y alteraciones en la comunicación (Heredia \& Durán, 2013). El deporte puede ser un pilar clave para alcanzar un desarrollo total en los ámbitos personales y sociales (López, Moreno \& López, 2020).

Los estudios cuyo fin es atender las necesidades educativas especiales sitúan su foco principal de atención en las personas con discapacidad física o sensorial, y no tanto a las personas con discapacidad psíquica o Trastornos Generales del Desarrollo (Heredia \& Durán, 2013). Además, el número de investigaciones científicas sobre programas de intervención hacia las personas con discapacidad a través del deporte no es elevado (Rello \& Garoz, 2014). Esto es un doble problema pues, por un lado, los principios de inclusión y atención a las personas con discapacidad a través del deporte y la actividad física han de sostenerse, de manera obligatoria, en métodos científicos (De la Caridad, Sailema, Rodríguez, Espinosa, Bayas \& Gibert, 2017). Por otro lado, el desarrollo de investigaciones científicas relacionadas con el deporte, diversidad funcional e inclusión lograrían fundamentar procesos metodológicos que ayudasen a la transformación de la realidad y contexto existente (De la Caridad et al., 2017).

Los patrones que caracterizan el espectro autista (comportamiento, intereses y actividades restrictivas y repetitivas, limitaciones en las interacciones sociales y comunicación) interfieren en las oportunidades de práctica de la actividad físico-deportiva, debido a la dificultad para jugar de forma imaginativa, participar en juegos sociales, hacer amigos, etc. Esto trae en conse- 
cuencia una predisposición hacia la inactividad y el ocio sedentario de las personas con autismo (Ruiz, Ponce, Sanz \& Valdemoros, 2015). Todas las personas con TEA poseen una gran necesidad de práctica y desarrollo de las capacidades motoras, deterioradas a consecuencia de la dificultad sostenida para estatuir mecanismos propios para su progreso (Ramírez et al., 2016). También, aun no siendo el déficit en las habilidades motrices básicas un criterio diagnóstico delTEA, es habitual su aparición en dicho trastorno (López, Moreno \& López, 2020). Es creído que la motricidad no se ve directamente afectada por el TEA, pero sí lo es de manera indirecta, pues las patologías neurológicas afectan al perfil neuromotor de las personas con autismo (Sánchez et al., 2019). Es usual que las personas con autismo no consigan los hábitos recomendados de actividad física, por lo que es esencial que este colectivo tenga las oportunidades necesarias para la práctica físico-deportiva, pues este mismo hecho (las oportunidades) son la barrera más limitante (Ruiz et al., 2015). Las personas con autismo tienen derecho al juego, deporte y tiempo libre, pero éste se ve dificultado, además de por las oportunidades, por la falta de tiempo y por otros obstáculos totalmente reversibles como la falta de formación de los profesionales, que no están capacitados en demasiadas ocasiones para el trabajo con personas con TEA (Klein, 2019).

La actividad físico-deportiva actúa en diversos focos, como son el terapéutico, educativo, competitivo y recreativo (Sánchez et al., 2019). Por ello, en las escuelas, la educación física es un medio muy positivo de intervención terapéutico para personas con TEA, pues ejerce como herramienta de mejora en la capacitación psicomotriz y adaptación e inclusión (Fernández, 2011), sin obviar su función catártica donde el alumno puede soltar toda su energía (Sánchez et al., 2019). Siguiendo la línea educativa trazada, la integración del ejercicio físico en el ámbito escolar mejora el comportamiento y conducta de los niños con TEA (Suárez et al., 2019) dentro del área de la educación física y también en patios y recreos, pues los niños con autismo muestran en estos espacios gran inactividad (Ruiz et al., 2015). Es importante el centro escolar como transmisor deportivo, pues es una manera directa de crear adherencia a la práctica físico-deportiva en la niñez, etapa vital para la adquisición de este hábito (Suárez et al., 2019). Además, dentro del deporte es reseñable el concepto del «juego», pues la experiencia lúdica es un acto en sí mismo, no es una recepción de un contenido ajeno, sino una vivencia donde receptor y juego son bidireccionalmente nutridos (Klein, 2019).
El deporte es un factor que contribuye en la mejoría de la población con autismo en procesos sociales y motores. Es una herramienta potencial para el desarrollo personal, social y psicológico de la población (Ramírez et al., 2016). El efecto del deporte en las personas con autismo también es relajante: minimiza los niveles de cortisol, descendiendo los niveles de ansiedad y estrés, lo que supone la mejora en la calidad del sueño y el humor, hecho que directamente afecta a la mejora del comportamiento de las personas con autismo (Suárez et al., 2019). La práctica de actividad físico-deportiva a intensidad moderada-vigorosa produce un aumento del nivel de noradrenalina y dopamina en el encéfalo que produce mejoras en la actividad cerebral, repercutiendo de manera directa en las funciones ejecutivas (Suárez et al., 2019). El deporte supone mejoría en las habilidades motoras básicas como coordinación, equilibrio y flexibilidad (Ramírez et al., 2016). Ejerce un efecto beneficioso en la propia percepción de competencia de las personas, asumiendo altos niveles de autoeficacia cuando poseen gran confianza en sus habilidades a la hora de hacer una tarea concreta, siendo así un aspecto vital en la conducta adaptativa de la persona con el entorno (Tejero et al., 2016).

El deporte constituye una vía esencial para el logro de la inclusión en las personas con discapacidad (De la Caridad et al., 2017). El hecho deportivo ha de ser visto desde una perspectiva donde la persona con discapacidad que realiza la práctica deportiva no es un ser pasivo (receptor de adaptaciones y asistencia que ayuden a su accesibilidad al mundo deportivo) sino activo, contribuyendo al crecimiento y posicionamiento de su colectivo (Asenjo \& Caro, 2019). En este sentido, hemos de atender al efecto producente o contraproducente del contexto en el desarrollo de las capacidades potenciales de las personas (Tejero et al., 2016).

\section{El baloncesto y el TEA}

\section{Progresión histórica del baloncesto en materia de inclusión}

La historia de las personas con discapacidad nace desde la discriminación, exclusión y persecución; si bien esta historia ha progresado hacia una perspectiva de derecho, ya que aún mantiene ciertas características que hacen perdurar en la mentalidad de la sociedad la discapacidad como pecado o enfermedad (Klein, 2019).

El baloncesto atañe a una construcción social encuadrada en el ámbito educativo, pues fue creado con una finalidad puramente pedagógica (Mujica, 2019). James 
Naismith, profesor de un centro universitario católico en Estados Unidos, inventó este deporte en 1891 tras la demanda del director de crear un juego con dos condiciones: la práctica debía ser a campo cubierto y representar los valores cristianos (Barthe, 1992). Por consiguiente, se sitúa el origen del baloncesto en la percepción emocional del alumnado (Mujica, 2019) por varias razones: el centro universitario abarcaba a jóvenes etiquetados como «incorregibles» (Barthe, 1992) y los estudiantes tenían falta de interés por las actividades que no fuesen al aire libre, por lo que la práctica de baloncesto tiene su meta en lograr ser una actividad que genere emociones positivas en el alumnado en las clases de educación física del centro (Mujica, 2019). De hecho, James Naismith se inspiró en un juego popular de su infancia para inventar el baloncesto, llamado «el pato en la roca» y que evocaba el disfrute y entretenimiento en sí mismo (Barthe, 1992).

La ideología humanista intrínseca en su creación hace al baloncesto un agente educativo, recreativo y deportivo (Mujica, 2019), inventado también para lograr evadir conflictos y situaciones agresivas generadas en otros deportes más populares como el fútbol o el rugby (Barthe, 1992). El baloncesto puede verse como un deporte americano que logró su expansión por todo el mundo (Mujica, 2019), envuelto en las características de fino y educado. Su práctica se extendió a las mujeres, cuando aún el género femenino no tenía acceso a la educación físico-deportiva como los hombres (Barthe, 1992). Este hecho supuso la catalogación del baloncesto como deporte afeminado, que produjo rechazo por parte de población masculina (Mujica, 2019).

El juego se introdujo con trece reglas concretas, que memorablemente no especificaban el número de jugadores por equipo, el campo de juego no tenía delimitación (era tan grande como el espacio en el que se llevase a cabo su práctica) y durante los tres primeros años del deporte su práctica se llevaba a cabo con balones de fútbol (Barthe, 1992). Como se ha señalado anteriormente, se pretendía que el baloncesto llevase como identidad el ser un juego esencialmente no violento, basados en el desarrollo humano y orientado hacia la perfección moral (Mujica, 2019). Esta imagen pulcra del baloncesto tuvo sus baches, pues tras varios incidentes en partidos cuando su práctica comenzaba a ganar popularidad, se crearon alambradas que rodeaban los recintos de juego para proteger a los jugadores de los objetos que arrojaban los espectadores, también los árbitros llevaban un arma reglamentaria (Barthe, 1992).

Se puede considerar que el baloncesto, desde sus inicios, siempre ha sido un deporte inclusivo (Mujica, 2019) y recreativo, pues fue su propio creador quien quiso etiquetarlo como deporte de exhibición y desestimaba la figura de un entrenador deportivo (Barthe, 1992). En este sentido, cabe destacar que la competición en la práctica deportiva puede ser advertida como un lastre en el camino hacia la inclusión, es propensa a tomar una dirección negativa al no ofrecer igualdad de estatus en los practicantes y no tener un objetivo común (Pérez-Tejero et al., 2012).

A lo largo de los años, se busca mantener el espíritu deportivo del baloncesto y se prohíbe su práctica en almacenes y salas de baile (Barthe, 1992). Por otro lado, el deporte comienza a popularizarse y a expandirse por todos los rincones del mundo. En la primera década del S.XX en España no alcanza apenas popularidad, siendo un deporte ignorado y con pocos practicantes (Mujica, 2019); en los periódicos se ponían anuncios para buscar jugadores y jugadoras para los clubes que, poco a poco, iban generándose (Barthe, 1992). El gobierno franquista en España (1939-1975) supuso un antes y un después en el deporte en este país, pues se ejerce un excesivo control sobre el mundo deportivo con el que la población pierde autonomía en sus intereses y se centra en la práctica deportiva y de la educación física como medio de adoctrinamiento de nuevas generaciones (Mujica, 2019). La educación física se encontraba dividida en masculina (elemento militarista) y femenina (elementos higienistas y sexistas); el baloncesto fue considerado modalidad femenina, sin embargo, las posibilidades económicas, políticas y sociales- eran ventajosas para el género masculino (Mujica, 2019).

El baloncesto a lo largo de los años comienza a provocar interés en toda la población, lo que genera un gran impulso que termina por situar a nuestro deporte en una dinámica sociocultural caracterizada por los valores que forman una cultura deportiva con rasgos como la igualdad, la justicia y el progreso (Mujica, 2019).

Actualmente, es uno de los deportes de equipo más practicado por las personas con discapacidad intelectual (Pérez-Tejero, Pinilla \& Vanlandewijck, 2010). Se recogen a su vez muchas propuestas de baloncesto inclusivo para personas con discapacidad motórica; ejemplos de todos ellos son programas como el Campus Inclusivo de Baloncesto, organizado por la fundación Real Madrid (Pérez-Tejero et al., 2012), «Unifield Sports» derivado de Special Olympics (Rello \& Garoz, 2014), el Campus Recreativo de baloncesto de Gillespie (Pérez-Tejero et al., 2012) o el Programa Educativo Deporte Inclusivo en la Escuela (DIE) (Ocete \& Pérez, 2019). 
Sin embargo, el baloncesto no es un deporte asociado al TEA, pues se declina hacia deportes individuales alegando en ellos mayores beneficios para la persona (Massion, 2006). La presente revisión pretende transformar esta idea, demostrando que los deportes colectivos no son recomendaciones, sino verdaderas necesidades en la población con TEA; el baloncesto es el deporte ejemplar.

\section{Beneficios del baloncesto: transformación y mejora}

El deporte puede ser considerado como un lugar de aprendizaje social de las normas de comportamiento y valores cívicos de nuestra sociedad (Drobnic et al., 2013). Por ello puede y debe mostrar más funciones que el entretenimiento, es un contribuyente directo a la inclusión en la sociedad dentro de su estado de diversidad (Moscoso \& Muñoz, 2012). Los beneficios son múltiples, se evidencia que a través del deporte se propicia mejoría en el desarrollo social y motor, íntimamente relacionados en el proceso; a nivel social, es reseñable la mejora en la autodeterminación, relaciones sociales, interpersonales y emocionales (Ramírez et al., 2016). Los valores que transmite el deporte son indisolubles de un concepto tan abstracto como valioso e inefable: la felicidad (Drobnic et al., 2013). El deporte en la vida de las personas ha de liberar, estar lleno de movimiento, goce y placer, sin estar sugestionado a ningún tipo de presión o condicionante tóxico en la práctica.

Estas mejoras son más latentes en los deportes colectivos, pues las actividades cooperativas se basan en el trabajo en equipo, donde todas las personas han de implicarse en el aprendizaje del resto del grupo, formando el éxito la contribución grupal de todos los componentes (Jiménez, Ocete \& Pérez, 2018).

En el caso del baloncesto, se habla de un deporte donde todas las acciones motoras tienen tres fases: percepción, decisión y ejecución (Cañadas \& Ibáñez, 2010). Si bien existe un gran peso táctico (estrategias dentro del juego para conseguir un objetivo -encestar-) dentro del deporte y de tomas de decisiones, se van a ver influenciadas por índoles técnicas, físicas y, por último, pero no menos importante, psicológicas (Cárdenas \& Alarcón, 2010). Este aspecto es vital para el trabajo de aspectos perceptivos, decisionales y de ejecución en relación con las acciones motoras anteriormente mencionadas que confrontan el juego (Cañadas \& Ibáñez, 2010). En el caso de la etapa infantil el hecho es aún más relevante: valorar las percepciones con el foco en la niñez es básico para facilitar su motivación por la práctica deportiva (López \& Arias, 2019). Sin duda, estos elementos son evidencias directamente relacionales a los beneficios que podría suponer esta práctica deportiva a las personas conTEA, pues es una herramienta muy potente en cuanto al trabajo y refuerzo de las funciones ejecutivas, entre muchos otros aspectos positivos existentes.

El baloncesto es un deporte que está regido por una lógica interna que define la relación entre los componentes de un grupo y la interacción con el medio (Cárdenas \& Alarcón, 2010). A su vez, estas relaciones están sujetas a la situación personal de cada individuo, pues a lo largo de la práctica deportiva las personas involucradas en el juego sienten distintas emociones tintadas por experiencias, metas y evaluación del entorno; las emociones generadas a través del baloncesto son positivas, especialmente por el desarrollo de relaciones interpersonales basadas en el respeto, amistad, progreso en los aprendizajes y cohesión grupal (Mujica, Orellana \& Concha, 2018). Es pertinente incidir en las emociones por una doble causa: su impacto en el deporte (Mujica, 2018) y la sensibilidad de las personas con TEA hacia el ámbito emocional (Reyna, 2011).

Si se habla del impacto en el deporte, las emociones gozan de un espacio diferencial en la construcción del aprendizaje motriz, influyendo en procesos cognitivos, funciones ejecutivas y aspectos motivacionales (Mujica, 2018). Emociones y pensamientos se nutren entre ellos (Mujica, 2018) lo que recalca la importancia del logro e impacto positivo de, en este caso, el baloncesto, en las personas; dentro de esta realidad, las personas con TEA focalizan su atención emocional influenciados de manera directa por factores situacionales (Reyna, 2011). De nuevo aparece en el mapa la importancia del entorno y contexto en que se adecue la práctica deportiva.

Por otro lado, se debe tener en cuenta que la ventaja del baloncesto más destacada no sólo por profesionales deportivos, sino también por pedagogos y docentes, es su alto valor cooperativo (Torrebadella, 2014). La práctica deportiva en la niñez va mucho más allá del alcance de habilidades específicas: el beneficio es el logro de una auténtica educación integral (Ortega, Robles, Abad, Durán, Franco, Jiménez \& Gimenez, 2018). El baloncesto debe ser un aliado directo de la inclusión, abriendo su mirada a un enfoque educativo; es un deporte lleno de potencial en la formación del alumnado, atendiendo a las dimensiones cognitivas, motriz y afectivosociales (Mujica, 2019). Además, el origen histórico del baloncesto revisado en anteriores puntos del trabajo si- 
túa al deporte como contribuidor para erradicar actitudes y conductas violentas en las personas (Mujica, 2019), teniendo consigo todos los ingredientes éticos, higiénicos y morales extrapolables directamente al contexto escolar (Torrebadella, 2014). En esta línea, la igualdad de oportunidades y posibilidad deportiva para las personas con discapacidad, al igual que para otros colectivos a priori minoritarios o excluidos -por ejemplo, desde la perspectiva ética, racial o de género- podría ser una realidad si se potencia en la práctica deportiva el desarrollo personal integral de las personas, empoderado y reconocido en méritos de manera igualitaria (Iglesia, Lozano \& Manchado, 2013).

Como señalan Asenjo \& Caro (2019. p.369) «el deporte inclusivo nos enseña que, se exteriorice o no, se llenen o no estadios, se logren o no cuotas ingentes de ganancia, la autosuperación es el mayor mérito y, al menos moralmente, merecerá siempre el mayor respeto».

\section{Discusión y conclusiones}

El objetivo del trabajo es revisar qué dicta la literatura en relación al TEA con el deporte, añadiendo el baloncesto como deporte a examinar, dada su posible potencialidad en el desarrollo integral de personas con autismo. La accesibilidad es crucial para realizar cualquier tarea del día a día; la actividad física y el deporte también requieren esa posibilidad (Marín, De Barros \& Hernández, 2020). Se ha evidenciado que deporte y diversidad funcional se nutren de manera bidireccional, alegando sus beneficios a nivel social, emocional, personal, siendo transmisor de hábitos saludables y mejora en habilidades sociales (Bernate et al., 2020; De la Caridad etal., 2017; Fernández, 2011; López, 2020; Massion, 2006; Moscoso, 2012). Es fundamental entender las posibilidades de las personas con diversidad funcional, teniendo como fin la creación de experiencias deportivas de calidad (Marín et al., 2020). En el caso de las personas con TEA, un aspecto principal a la hora de realizar la práctica deportiva es la accesibilidad cognitiva: entender el entorno para poder interactuar con él; cuidar los campos de gran impacto en la actividad cerebral, generando buenos hábitos de vida. A lo largo del artículo quedan recogidas las características principales que acentúan el diagnóstico delTEA, adherido no sólo a un corte biológico sino también psicológico. ElTEA se manifiesta de manera muy distinta en cada persona -es difícil atribuirle unas características concretas, pues a pesar de ser coincidente la patología, su desarrollo difiere se- gún el sujeto- (Heredia \& Durán, 2013). Por ello, es importante actuar diferente con cada persona con autismo (Sánchez et al., 2019). Esta personalización ha de sostener el corte psicológico asociado como un básico dentro del desarrollo de la persona. Este hecho, de nuevo, nos lleva a la idea de la facilitación de ambientes (Heredia \& Durán, 2013) para el desarrollo más óptimo e integral posible. El enfoque ha de ser sistémico, contemplando que se adecue a la «triada del autismo»: deficiencias en la interacción social; alteración en la comunicación; patrones de comportamiento, actividades restringidas, repetitivas y estereotipadas (García, Alpizar \& Gúzman, 2019). Dicha adecuación hace seguros los beneficios en la mejora en la regulación emocional y estado de ánimo, entre otros (Bernate et al., 2020). La sociedad se encuentra en un momento de re-construcción inclusiva donde se ha comprendido que no se encontraba diseñada para todos; en otras palabras, el contexto sostiene déficits que es tiempo de erradicar, teniendo todas las personas los mismos derechos y oportunidades.

De igual manera, se fundamenta el baloncesto como un deporte históricamente inclusivo donde podemos encontrar una posibilidad de trazado bidireccional con el TEA, retroalimentándose de las características del baloncesto que compactan tan bien con las mayores dificultades que muestra el diagnóstico del trastorno. El origen histórico del baloncesto sitúa al deporte como contribuidor directo de la inclusión (Mujica, 2019). La enseñanza deportiva es un proceso continuo de toma de decisiones del docente y del discente: por un lado, las acciones pedagógicas del entrenador y/o profesor; por otro lado, el predominio de acciones colectivas ofensivas y defensivas (Gamero, García-Ceberino, Reina, Feu \& Antúnez, 2020) que invitan continuamente a actuar en función del juego. El baloncesto produce sensaciones y emociones positivas, muy vinculadas al desarrollo interpersonal -respeto, amistad, progreso en los aprendizajes, cohesión grupal- (Mujica, Orellana \& Concha, 2018), teniendo un alto valor cooperativo (Torrebadella, 2014) que le convierte en un tesoro y vía de logro hacia la inclusión. Son escasos los estudios que aborden el baloncesto desde la percepción emocional (Mujica \& Jiménez, 2020). Sáenz-López, Duque, Almaro \& Conde (2020) citan que el deporte es emocional y emocionante; son convenientes líneas futuras de investigación que sostengan estas palabras, ahondando en el estudio del baloncesto como medio sobresaliente de desarrollo e inclusión.

Como principales limitaciones destaca la escasa evi- 
dencia científica que relacione elTEA con el baloncesto. La falta de existencia de conocimiento científico deriva en múltiples dificultades. Uno de los problemas es la generalización en relación a los grados de TEA y actividad física, cuando hay grandes diferencias entre ellos; se atiende elTEA como un diagnóstico único, olvidando la actuación individualizada que ha de realizarse con cada persona con TEA (Sánchez et al., 2019), sumado al misterio que aún esconde el origen y naturaleza del trastorno. Además, se entremezclan enfoques escolares, extraescolares y deportivos que complican la generalización científica (Gamero et al., 2020). El objetivo a perseguir es desarrollar valores fundamentales de la vida de las personas, evitando que los esfuerzos sean orientados a luchar contra la sintomatología (Lozano et al., 2017). Una de las consecuencias de observar elTEA con forma única, en vez de poliédrica, es la generalización que, en este caso, puede afectar a la concepción de los deportes colectivos. El mayor foco de estudio se encuentra localizado en actividades físicas individuales, hidrogimnasia, equinoterapia, carrera, juegos recreativos y bicicleta fija (Fessia, Manni, Contini \& Astorino, 2018). El baloncesto, deporte muy completo en cuanto a beneficios personales-sociales-saludables (Bretón \& Castro, 2017), queda fuera de la ecuación de estudio TEA-deporte al estar en el escenario de deportes colectivos, aún sin suficientes estudios. A su vez, hubiese sido muy positiva la incorporación de experiencias y trabajos de campos anteriores en dicha relación (TEA y deportes colectivos). Actualmente, la mayoría de estudios que relacionan el baloncesto con la diversidad funcional se inclinan por la discapacidad motórica (Gil, 2011; Pérez-Tejero et al., 2012; Santana \& Garoz, 2013). Es por ello que la mayoría de programas científicamente recogidos se encuentran apegadas a dicha característica.

Como líneas de propuesta futuras, la revisión narrativa es una oportunidad y una reflexión para contabilizar y explorar los deportes colectivos como positivos y beneficiosos para las personas con autismo, y como medio idóneo de inclusión social. El deporte es una fuente de vida y un gran aliado en la apuesta por el desarrollo integral de las personas; su práctica y disfrute no ha de ser un privilegio, sino un derecho para todas las personas, sin excepción. Para abarcar este camino, es necesaria la inclusión total de la sociedad -inversión en investigación, en políticas, en educación-, atendiendo al deporte como medio potencial de desarrollo integral de las personas con diversidad funcional. Otro paso obligado es la necesaria fundamentación del TEA en relación con los deportes colectivos, desde una perspectiva que se centre en la potencialidad, no en la «limitación» (no es las persona quien las posee, es el entorno y sus barreras globales). Lo diferente no es una limitación, es un enriquecimiento, una oportunidad. El baloncesto tiene un recorrido histórico que avala su potencial inclusivo y repercute de manera incisivamente beneficiosa en competencias interpersonales e intrapersonales de sus practicantes; merece la pena ahondar en su estudio y su posible relación con el TEA.

Tras la revisión, se pueden abordar las siguientes conclusiones. Por un lado, la existencia de desigualdad en las oportunidades y oferta deportiva para las personas con discapacidad. Estas desigualdades sugieren su origen en estereotipos y creencias derivadas de la tradición sociocultural arraigada en la sociedad hacia la práctica deportiva de personas con TEA. Por otro lado, se realzan los deportes colectivos, específicamente el baloncesto, como deporte a estudiar y valorar en relación a los beneficios que puede generar en las personas con TEA, exprimiendo todo su potencial a nivel social, físico, cognitivo y educativo. Por último, realzar la necesidad y compromiso urgente de la sociedad por alcanzar la inclusión en todos sus ámbitos.

\section{Referencias}

André, T.G., Valdez, C., Ortiz, R.E. \& Gámez, M.E. (2020). Prevalencia del trastorno del espectro autista: una revisión de la literatura. Jóvenes en la Ciencia, 7, 1-7.

Asenjo, F. \& Caro, J. (2019). Inclusión y competitividad en el deporte federado de personas con discapacidad. Revista Española de Educación Física y Deportes -REEFD, 426, 362369.

Barthe, P. (1992). Orígenes del baloncesto. Barcelona (España). RTVE.

Bernate, J., Rojas, M., Fonseca, I., Betancourt, M.J. \& Urrea, P. (2020). Estrategia pedagógica de actividad física planificada en autismo: Sistematización de experiencia. Acción Motriz, 25, 48-58.

Braga, G. (2011). O esporte como elemento socializador e formador de crianças e jovens. Revista Científica da Faminas, 6(2), 126-140.

Bretón, S. \& Castro, M. (2017). Adolescencia y baloncesto. Journal of Sport and Health Research, 9(1), 97-108.

Cañadas, M. \& Ibáñez, S.J. (2010). La planificación de los contenidos de entrenamiento de baloncesto en equipos de iniciación. E-balonmano, Revista de Ciencias del Deporte, 6(1), 49- 56.

Caracuel, J. \& Arbinaga, F. (2012). Repercusión del ejercicio físico sobre la salud. Apuntes de psicología, 30(1-3), 547554.

Carbonero, L. \& Prat, M. (2012). Las familias y la promoción 
del deporte en edad escolar. Padres y Madres de Alumnos y Alumnas. Revista de la Confederación Española de Asociaciones de Padres y Madres de Alumnos (CEAPA), 113, 16-19.

Cárdenas, D. \& Alarcón, F. (2010). Conocer el juego en baloncesto para jugar de forma inteligente. Walceulen $E F$ Digital, 6, 52-72.

Chamero, M. \& Fraile, J. (2013). Relación del disfrute en la actividad físico-deportiva con la autoeficacia motriz percibida al final de la infancia. Revista Qurriculum, 26, 177196.

De la Caridad, G., Sailema, A., Rodríguez, A., Espinosa, Y., Bayas,A. \& Gibert,A.R. (2017). Experiencias investigativas en el contexto de la actividad físico-deportiva y recreativa adaptada e inclusiva. Educación Física y deportes, 226, 1-14.

Drobnic, F., García, A., Roig, M., Gabaldón, S., Torralba, F., Cañada, D., ... Prat, F. (2013). La actividad física mejora el aprendizaje y el rendimiento escolar. Los beneficios del ejercicio en la salud integral del niño a nivel físico, mental y en la generación de valores. Esplugues de Llobregat (Barcelona): Hospital Sant Joan de Déu.

Felipe Rello, C., Garoz Puerta, I., \& Tejero González, C. M. (2018). Análisis comparativo del efecto de tres programas de sensibilización hacia la discapacidad en Educación Física. Retos, 34, 258-262. https://doi.org/10.47197/ retos.v0i34.59889

Fernández,A., \& Camargo, C. (2021). Inclusión, atención a la diversidad y neuroeducación en Educación Física. Retos, 41, 555-561. https://doi.org/10.47197/ retos.v0i41.86070

Fernández, J. (2011). La actividad físico-deportiva como base para la integración en el área de educación física: autistas. EmásF, Revista Digital de Educación Física, 8, 24-31.

Fessia, G., Manni, D. , Contini, L. \& Astorino, F. (2018). Estrategias de actividad física planificada en autismo: revisión sistemática. Revista de Salud Pública, 20(3), 390-395. doi:10.15446/rsap.v20n3.63040

Gamero, M.G., García-Ceberino, J.M., Reina, M., Feu, S. \& Antúnez, A. (2020). Estudio de las variables pedagógicas de las tareas de baloncesto en función de las fases de juego. RETOS. Nuevas tendencias en Educación Física, Deporte y Recreación, 37, 556-562.

García, A., Alpizar, O.A. \& Guzmán, G. (2019). Autismo: revisión conceptual. Boletín científico de la Escuela Superior de Atotonilco de Tula, 6(11), 26-31. doi:10.29057/ esat.v6i11.3693

García, D. \& Polaino, A. (2011). El autismo y las emociones: nuevos hallazgos experimentales. Valencia: Promolibro.

Gil, A.M. (2011). Avances en la investigación del deporte para personas con discapacidad. Archivos de Medicina del Deporte, 28, 165-167.

Heredia, J. \& Duran, D. (2013). Aprendizaje cooperativo en educación física para la inclusión de alumnado con rasgos autistas. Revista nacional e internacional de educación inclusiva, 6(3), 25-40.
Hernández, O., Risquet, D., Hernández, O., León, M., Pérez, A. \& Ballate, D. (2016). La intersectorialidad en la atención a niños y adolescentes con trastornos del espectro autista. Acta Médica del Centro, 10(2), 8-16.

Hernández, V.A., Calixto, B. \& Aguilar, I.E. (2012). Aspectos psicológicos de familiares de personas diagnosticadas con Trastorno del Espectro Autista (TEA). Revista Intercontinental de Psicología y Educación, 14(1), 73-90.

Iglesia, M., Lozano, I. \& Manchado, L. (2013). Deporte e igualdad: las voces de las deportistas de élite. Feminismo/ $s, 21,71-90$.

Jiménez, A., Ocete, C. \& Pérez, J. (2018). Las actividades cooperativas como herramienta de inclusión de alumnado con discapacidad en educación física: el programa «deporte inclusivo en la escuela». XI congreso Internacional de Actividades Físicas Cooperativas (pp.959-968). Oviedo: Ediciones de la Universidad de Oviedo.

Klein, S. (2019). Juego y autismo: el lugar que ocupa lo recreativo en la vida social de las personas con TEA desde una perspectiva de derecho. XII Jornadas de Sociología. Facultad de Ciencias Sociales, Universidad de Buenos Aires, Buenos Aires.

Latorre Román, P., Sánchez Salvador, M., Salas Sánchez, J., \& García Pinillos, F. (2018). El nivel bajo de la aptitud física es una característica temprana en niños preescolares con autismo. Retos, 35, 348-350. https://doi.org/ $10.47197 /$ retos.v0i35.58052

López-Herrero, F., \& Arias-Estero, J. L. (2019). Efecto de la modalidad de juego en baloncesto ( $5 \mathrm{vs.} 5$ y $3 \mathrm{vs} .3$ ) sobre conductas motrices y psicológicas en alumnado de 9-11 años. Retos, 36, 354-361. https://doi.org/10.47197/ retos.v36i36.67163

López, J.M., Moreno, R. \& López Bastías, J.L. (2020). Análisis del impacto de un programa deportivo en niños con Trastorno del Espectro del Autismo. Retos, 39, 98-105.

López, M. (2011). Barreras que impiden la escuela inclusiva y algunas estrategias para construir una escuela sin exclusiones. Innovación Educativa, 21, 37-54.

Lozano, M.C., Manzano,A., Casiano, C. \& Aguilera, C. (2017). Propuesta de intervención en familiares de niños conTEA desde ACT para mejorar la convivencia familiar y escolar. Asociación Nacional de Psicología Evolutiva y Educativa de la Infancia, adolescencia y mayores, 1(1), 45-55.

Marín-Perabá, C., De Barros-Camargo, C., HernándezFernández, A. (2020). La inclusión de calidad desde el punto de vista de la educación física y la discapacidad. Journal of Sport and Health Research, 12(3), 271-284.

Massion, J. (2006). Sport practice in autism. Science \& Sports, 21(4), 243-248. doi:10.1016/j.scispo.2006.07.001

Moreno, J.A., Águila, C. \& Borges, F. (2011). La socialización en la práctica físico-deportiva de carácter recreativo: predictores de los motivos sociales. Apunts Educación Física y deporte, 103, 76-82.

Moscoso, D. \& Muñoz,V. (2012). Deporte, inclusión y diver- 
sidad social, antecedentes. Anduli: Revista Andaluza de Ciencias Sociales, 11, 13-19.

Mujica, F.N. \& Jiménez, A.C. (2020). Percepción emocional del alumnado de $3^{\circ}$ ESO ante las prácticas de la unidad didáctica del baloncesto en educación física. Revista Española de Educación Física y deportes-REEFD-, 429, 47-60.

Mujica, F.N., Orellana, N.C., \& Concha, R. (2018). Atribución emocional en el taller de baloncesto escolar de una escuela pública en Chile: análisis de contenido. Cuadernos De Psicología Del Deporte, 18(1), 31-42.

Mujica, F.N. (2018). Las emociones en la educación física escolar: el aporte de la evaluación cualitativa. EmásF: Revista Digital de Educación Física, 51, 64-78.

Mujica, F.N. (2019). El sentido moral que James Naismith otorgó al baloncesto: una fortaleza para su desarrollo en España y en la Educación Física. EmásF, Revista Digital de Educación Física, 56, 92-103.

Muñoz, E.M., Garrote, D. \& Sánchez, C. (2017). La práctica deportiva en las personas con discapacidad: motivación personal, inclusión y salud. Revista INFAD de psicología, 1 , 145-152. https://doi.org/10.17060/ ijodaep.2017.n1.v4.1037

Ocete, C. \& Pérez, J. (2019). Una experiencia en deporte inclusivo: el evento final del programa Deporte Inclusivo en la Escuela (DIE). Padres y maestros, 377, 30-34.

Ortega Vila, G., Robles Rodríguez, J., Abad Robles, M. T., Durán González, L. J., Franco Martín, J., Jiménez Sánchez, A., \& Giménez Fuentes-Guerra, F. J. (2018). Las preferencias de interacción social en las Escuelas Sociodeportivas de Baloncesto de la Fundación Real Madrid. Retos, 35, 101-106. https: / / doi.org/10.47197/ retos.v0i35.62992

Pelegrín, A., Garcés, E. \& Cantón, E. (2010). Estudio de conductas prosociales y antisociales. Comparación entre niños y adolescentes que practican y no practican deporte. Información Psicológica, 99, 64-78.

Pérez-Tejero, J., Ocete, C., Ortega-Vila, G. \& Coterón, J. (2012). Diseño y aplicación de un programa de intervención de práctica deportiva inclusiva y su efecto sobre la actitud hacia la discapacidad: el Campus Inclusivo de Baloncesto. Revista Internacional de Ciencias del Deporte, 8(29), 258-271. doi:10.5232/ricyde2012.02905

Ramírez, I., Negrete, D., Díaz, B., Rubiano, P. \& Hernández,T. (2016). Revisión sistemática de programas deportivos aplicados a personas con autismo. Revista Ciencia y Actividad Física, 3(2), 63-74.

Rello, C. \& Garóz, I. (2014). Actividad físico-deportiva en programas de cambio de actitudes hacia la discapacidad en edad escolar: una revisión de la literatura. Cultura, Ciencia y Deporte, 9(27), 199-210.

Reyna. C. (2011). Desarrollo emocional y trastorno del espectro autista. Revista de Investigación en Psicología, 14(1), 273-280. doi:10.15381/rinvp.v14i1.2087

Robles-Rodríguez, J., Abad-Robles, M.T., Giménez Fuen-
tes-Guerra, J., \& Benito-Peinado, P. (2016). Los deportes adaptados como contribución a la educación en valores y a la mejora de las habilidades motrices: la opinión de los alumnos de Bachillerato. Retos, 31, 140-144. https:// doi.org/10.47197/retos.v0i31.49418

Ruiz, D., Salinero, J.J., González, C., Lledó, M., García, T., Theirs, C.I., Nombre, D. \& Guitián, A. (2015). Descripción de la práctica de actividad física, habilidades motrices básicas y composición corporal en niños y jóvenes del espectro autista. Diferencias por sexo. Retos, 28, 61-65. doi: $10.47197 /$ retos.v0i28.34817

Ruiz, J.V., Ponce, A., Sanz, E. \& Valdemoros, M.A. (2015). La educación en valores desde el deporte: investigación sobre la aplicación de un programa integral en deportes de equipo. Retos, 28, 270-275.

Sáenz-López, P., Duque, V.H., Almagro, B.J. \& Conde, C. (2020). Baloncesto y emociones: una revisión sistemática. E-balonmano: Revista de Ciencias del Deporte, 16(1), 7384.

Sánchez, J., Sánchez,A., Pastor, J.C. \& Martínez, J. (2019). La formación docente ante el trastorno del espectro autista. Revista Euroamericana de Ciencias del Deporte, 8(2), 5966. doi:10.6018/sportk.401121

Sánchez, M. \& Collado, J.A. (2016). Factores determinantes en la enseñanza-aprendizaje del deporte desde la perspectiva emocional. E-balonmano, Revista de Ciencias del Deporte, 12(2), 119-128.

Santana, P. \& Garoz, I. (2013). Actitudes hacia la discapacidad e intervención docente desde el deporte adaptado. Revista Internacional de Medicina y Ciencias de la Actividad Física y del Deporte, 13(49), 1-17.

Santos, P., Jiménez, J. \& Rioja, N. (2019). Efecto de un programa de ejercicio de corta duración sobre la condición física y la calidad de vida en mujeres supervivientes de cáncer de mama del ámbito rural: Estudio Piloto. RICYDE, Revista Internacional de Ciencias Del Deporte, 15(56), 171-186. doi: 10.5232/ricyde2019.05604

Suárez, S., López, S., Belchior, P. \& Da Cruz, L.M. (2019). El ejercicio físico en el aula para la mejora del comportamiento de niños autistas. Revista Iberoamericana de Ciencias de la Actividad Física y el Deporte, 8(1), 136-148. doi:10.24310/riccafd.2019.v8i1.5792

Tárraga, R. \& Sanz, P. (2018). ¿Qué estrategias de intervención funcionan en la educación de los niños con trastorno del espectro autista? Revisión de evidencias en la literatura científica. Reidocrea, 7, 279-287.

Tejero, C., De la Vega, R., Vaquero, M. \& Ruiz, R. (2016). Satisfacción con la vida y autoeficacia en jugadores de baloncesto en silla de ruedas. Revista de Psicología del Deporte, 25(1), $51-56$.

Torrebadella, X. (2014). Notas para la historia del centenario del baloncesto español. Un deporte escolar y popular para ambos sexos (1897-1938). E-balonmano, Revista de Ciencias del Deporte, 10(3), 177-198. 